\title{
IPS OBSERVATIONS AT STELAB FOR THE FRUITFUL COMING DECADE
}

\author{
K. ASAI, Y. ISHIDA, M. KOJIMA, K. MARUYAMA, H. MISAWA and \\ N. YOSHIMI \\ Solar-Terrestrial Environment Laboratory, Nagoya University, Toyokawa 442, Japan
}

\begin{abstract}
We have been carrying out solar wind measurements using the interplanetary scintillation (IPS) method. Our IPS observation system is operated at a frequency of $327 \mathrm{MHz}$ and consists of four stations located at Toyokawa, Fuji, Sugadaira and Kiso. The present system, however, has insufficient sensitivity to measure enough IPS sources for observing the solar wind with adequate spatial and temporal resolution. Therefore we have been excuting the upgrade project since 1994 in order to observe a larger number of compact radio sources. The Fuji system has been improved successfully and has achieved sensitivity by a factor over five compared with the previous system. The upgrade project is now in progress for the Toyokawa and Sugadaira station.
\end{abstract}

Key words: Solar wind - IPS - Upgrade project

\section{IPS Observations at STELAB}

The Solar-Terrestrial Environment Laboratory (STELAB) has been carrying out solar wind measurements using the interplanetary scintillation (IPS) method since the early 1970s (e.g., Kojima and Kakinuma, 1990). The IPS observations of the STELAB were started at a frequency of $69 \mathrm{MHz}$ using three stations located at Toyokawa, Fuji and Sugadaira (Fig. 1). At this frequency, however, the IPS technique cannot probe the solar wind at heliocentric distances within $0.3 \mathrm{AU}$ because radio scattering becomes strong. Although the solar wind measurements within 0.3AU are particularly important to study the acceleration processes of the solar wind, in situ measurements have not been made so far.

Therefore we shifted the frequency to $327 \mathrm{MHz}$ and built three parabolic cylinder antennas from 1977 to 1982 at the same sites where the $69 \mathrm{MHz}$ stations were located. In addition to these three antennas, one more antenna was constructed at Kiso in 1993, and since then, we have been making the IPS observations with a four-station system (Asai et al., 1994).

The antenna at each station is an asymmetric parabolic cylinder (Fig. 2) that is the same type as the Ooty radio telescope in India. Our antenna has a physical collecting area of about $2000 \mathrm{~m}^{2}$ and a long axis in the east-west direction. Each station is full-automatically operated and is connected to the host station (Toyokawa) with the public telephone line for data aquisition and system control. 

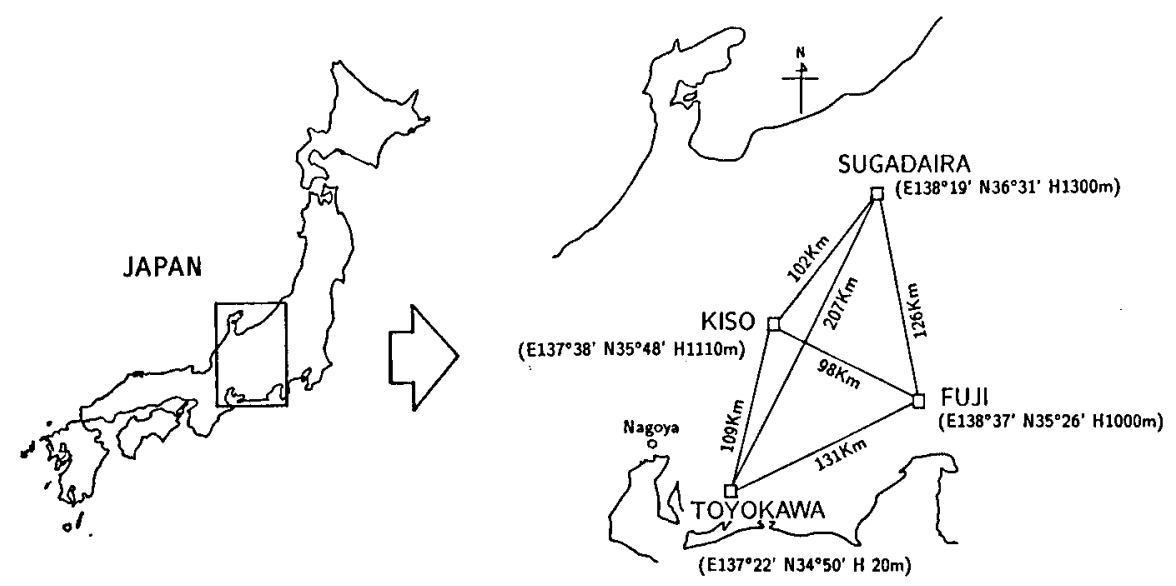

Fig. 1. Locations of four stations

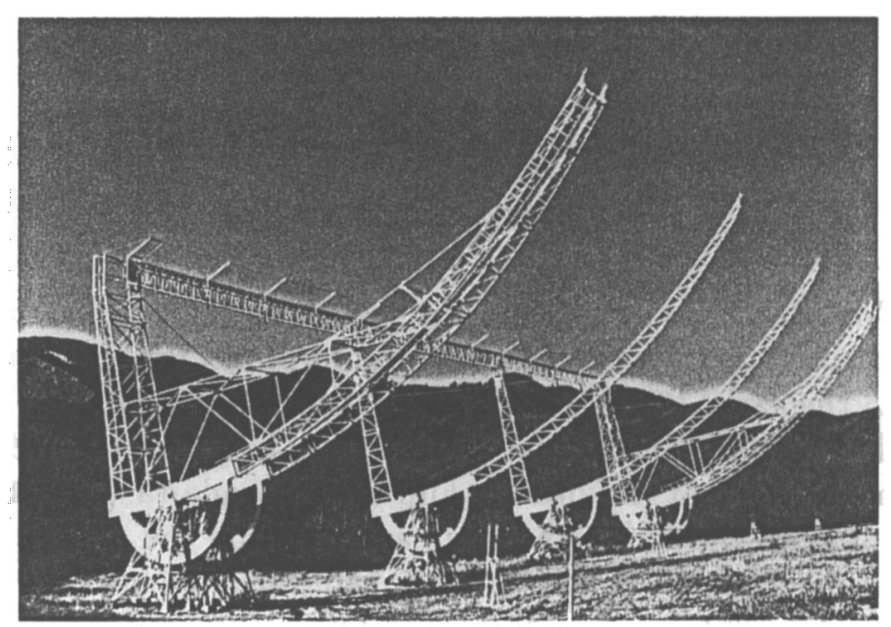

Fig. 2. UHF parabolic cylinder antenna at Kiso station

\section{Upgrade Project}

Now we are observing several tens of IPS radio sources every day. However, this is insufficient to produce one velocity-map during one solar rotaion. We also cannot measure the interplanetary transient phenomena with high resolution. Therefore we have been carrying out the upgrade project to observe a larger number of IPS radio sources.

Last year Fuji system was improved making the primary feed system designed optimumly with the same method as used for Kiso antenna (Asai et al., 1995), and installing low noise GaAsFET amplifiers to the feed system. The improved system achieved to be five times more sensitive than the previous 


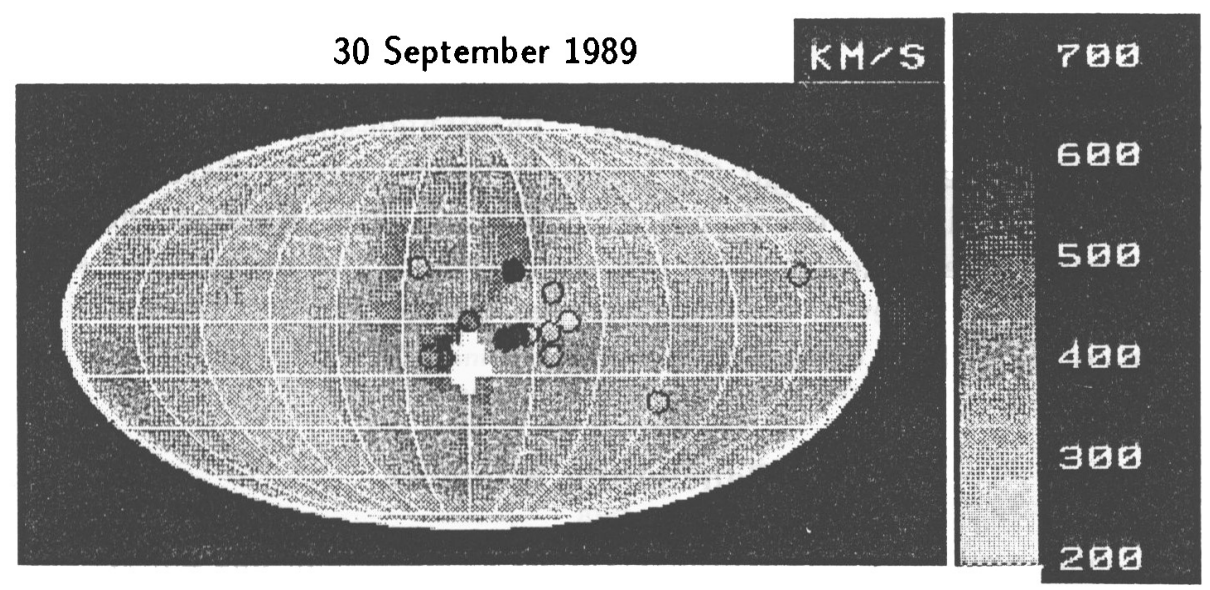

Fig. 3. Distribution chart of the solar wind speed deduced from our observations. The enhancements of the solar wind speed were observed on the next day of September 29, 1989 when the X-class flare occured at the west limb.

system. This project is now continuing for the stations of Toyokawa and Sugadaira.

\section{Result - Interplanetary Transient Phenomena}

We give an example of transient interplanetary disturbances obtained from our IPS observations. The IPS can catch interplanetary shocks and plasma clouds in association with coronal mass ejections or other solar activity as enhancements of the solar wind speed. The X-class flare occured at the west limb of the Sun on September 29, 1989. One day later, we measured the highspeed wind $(>700 \mathrm{~km} / \mathrm{s})$ due to the interplanetary disturbance associated with this flare event.

Figure 3 is a distribution chart of the solar wind speed taking a view on Earth. The Sun is located at the center of this Mercatorial coordinates. The horizontal axis is the ecliptic longitude, and the left and right edges correspond to the $-180^{\circ}$ and $+180^{\circ}$ directions from the Sun. The vertical axis is the earth centered latitude, and the upper and lower edges indicate the $90^{\circ}$ north and south directions from the Sun respectively. The circles in the charts represent the locations of the IPS radio sources on indicated day and the gray code corresponds to the speed. The background gray code indicates the background structure of the solar wind speed obtained by the following calculations. First, we make a synoptic map of the solar wind speeds by mapping back the IPS speeds onto the source surface, and superposing the data during four Carrington rotaions of the number from 1819 to 1822 . 
Second, the virtual IPS observations with a infinite number of IPS sources estimate the solar wind speeds projected into the interplanetary space.

The sudden enhancements of the solar wind speed were observed on the next day when the flare event occured. These high-speed winds could be evidence that the IPS caught the transient interplanetary disturbance associated with this flare event, not from coronal holes. Because the speed enhancements were measured in the region where the background shows the low speeds. However, unfortunately we cannot mention the details on account of lack of the observation resolution.

\section{Concluding Remarks}

When this upgrade project will be completed, more than one hundred IPS sources can be observed every day. This enables us to make the IPS observations with higher spatial and temporal resolution. As a result, we can predict to obtain fruits for the solar wind study as the followings. 1) The velocity estimate becames reliable. Moreover, 2) we can measure the interplanetary transient phenomena with higher resolution, which suggests that 3 ) it has potential to be used for the space weather forecast.

Besides this upgrade project, we plan to make the observations at two frequencies of $327 \mathrm{MHz}$ and the higher one so that the IPS can probe the near-sun regions. We also intend to move the Toyokawa station to the place in the better radio environment.

\section{Acknowledgements}

The authors wish to thank Dr. P. K. Manoharan and Dr. A. J. Selvanayagam for technical information and useful advice on the design of the preamplifier. The Asai's voyage to India for participating this IAU Colloquium No. 154 "Solar and Interplanetary Transients" was financially supported by the Satio Hayakawa Fund (Grant-in-Aid for young astronomers abroad) from the Astronomical Society of Japan.

\section{References}

Asai, K., Y. Ishida, M. Kojima, K. Maruyama, H. Misawa, and N. Yoshimi: 1994, 'Multi-station system for solar wind observations using the interplanetary scintillation method', J. Geomag. Geoelectr., in press.

Asai, K., M. Kojima, H. Misawa, Y. Ishida, K. Maruyama, N. Yoshimi, M. Wakasa, and M. Karakida: 1995, 'Design of an asymmetric parabolic cylinder antenna with high aperture efficiency', Trans. IEICE, J78, B-II, 102.

Kojima, M. and T. Kakinuma: 1990 , 'Solar cycle dependence of global distribution of solar wind speed', Space Sci. Rev., 53, 173. 